\title{
FLOW INTO AN ARTERIAL BRANCH MODEL
}

\author{
Mehran Tadjfar \\ Advanced Computing Center, \\ The Institute of Physical and Chemical Research (RIKEN) \\ 2-1, Hirosawa, Wako-shi, Saitama 351-0198 Japan \\ e-mail: mtadjfar@riken.go.jp
}

\begin{abstract}
Arterial branches are found to be a major site for formation of arterial plaque. In this study, we investigate several of the main parameters that influence the local flow into an arterial branch model. In particular, we are interested in the role of the local geometric parameters of the bifurcation on the overall flow. Specially, how the changes in the bifurcation geometry influence the distribution of axial wall shear and pressure in the model. The major geometric factors influencing this flow are the bifurcation area ratio and angle. The flow in a large number of geometric variations of our branch model is numerically simulated. The models at several branch area ratios in the range of $0.4 \leq A R \leq 2.0$ are considered. In the above range of area ratios, we also create various versions within a range of branch opening half-angle of $\pi / 25 \leq \theta \leq \pi / 4$. The flow in the above models is calculated for the inlet flow Reynolds numbers of 250, 500, 1000 , and 2000 .

Area ratio seems to have the largest influence on the flow within the physiologically relevant range of the parameters considered. Increasing the area ratio can lead to relatively large flow separation in the vicinity of the bifurcation region. At higher values of the opening angle of the bifurcation, the possibility and severity of flow separation at the appropriate wall location increases. The relative influence of the convective acceleration of the flow, as represented by the value of the flow Reynolds number is also investigated. The particular value of the area ratio or bifurcation angle, necessary to initiate flow separation, is influenced by the Reynolds number of the incoming flow in the mother tube. The relation between the influence of these parameters on the flow and the formation of some vascular diseases reported in the literature are also examined.
\end{abstract}




\section{Introduction}

In the human cardio vascular system many vascular diseases are found in the vicinity of vascular branches. Atherosclerosis is commonly found near regions of high curvature or bifurcation of large and medium size arteries. The tendency for plaque to form around and near the regions of vessel bifurcation is a well-known fact. Having the same general systematic risk factors for atheroma results in plaque formation around different arterial bifurcations for different individuals. In carotid bifurcations, with identical general risk factors in a given individual, we have asymmetry in the formation of carotid plaque. These seem to indicate the importance of the each bifurcation's unique geometry in plaque formation. Hence, we can hypothesize on the crucial role of local hemodynamic factors in plaque formation and its local distribution. Local hemodynamic forces undergo large changes in the vicinity of these regions due to the interaction of primary flow with the associated local secondary flow. Fluid mechanical forces directly influence endothelial cell structure and function on the vessel walls. The exact mechanisms of how the hemodynamic factors influence plaque formation and its further development are still subject to debate [1-5]. However, it is hypothesized that low wall shear stress regions and regions of high particle residence time are locations of high risk for vascular diseases. Hence, a better understanding of the hemodynamics of these local regions using CFD is important

There are many bifurcations in the arterial system with great variations in geometrical parameters within each one. Lou and Yang [6] in an excellent review of the bifurcation flow literature of that time summarize that the most important parameters influencing the flow into a branch are bifurcation angle, area ratio, the local wall curvature of the bifurcation region, and the flow waveform. They point out that the angle of aortic bifurcation, where the aorta bifurcates into two common iliac arteries, can vary between $10^{\circ}$ to $80^{\circ}$. Sharp et al. [7] in a review of abdominal and pelvic arteriograms of 100 patients divided the patients based on the relative level of the aortic bifurcation. In the 48 patients, whose aortic bifurcations were at or lower than the fourth lumbar vertebral resulting in a higher average bifurcation angle of $52^{\circ}$, only 7 patients (14\%) suffered from occlusive disease. In the remaining 52 patients with the aortic bifurcation above the L4 level with an average bifurcation angle of $38^{\circ}, 21$ patients $(40 \%)$ suffered from occlusive disease.

Karino and Goldsmith [8] in a set of careful flow visualizations studied the flow patterns and "vortex formation" (separation) in a model T-junction. They varied the branching angle and the side to main tube diameter ratio. They found that the diameter ratio (a measure similar to area ratio) is more influential on the flow. The effect of the branching angle on the flow separation in the main tube was much smaller. However, they reported an appreciable influence of the branching angle on the side tube separation.

Schulz and Rothwell [9] in a recent comprehensive study of natural variations in carotid bifurcation anatomy reviewed 5395 angiograms from 3007 patients. Amongst many parameters, they measured the following area ratios: 
internal to common carotid (ICA/CCA), external to common carotid (ECA/CCA), and the bifurcation total area ratio $(($ ICA+ECA $) / \mathbf{C C A})$. They found that the normal range of the bifurcation area ratio is between 0.38 to 1.28. Bifurcation anatomy also showed no systematic difference in any of their calculated parameters between left and right carotids within individuals. However, they reported considerable asymmetry within individuals in regards to ipsilateral and contralateral bifurcations to the stroke site. For example, they found that $42 \%$ of the patients had a side difference of $\geq 25 \%$ in the total area ratio of the bifurcation.

Friedman et al. [10,11] studied the side branches off of the left anterior descending coronary artery of 15 angiographically lesion-free human hearts obtained from autopsy. They found a strong positive correlation between intimal and medial thickening and large bifurcation angles. However they reported a weaker and more local correlation between the area ratio at the bifurcation site and the medial thickening. They also suggested that large branching angles are associated with more asymmetric thickening.

Fisher and Fieman [12] investigated the arteriograms of 40 male patients with carotid stenosis of less than $50 \%$. They measured stenosis diameter, (ICA/CCA) area ratio, and bifurcation angle in all those cases. They found significant association between diameter stenosis asymmetry and (ICA/CCA) area ratio asymmetry. However, there was no such association between stenosis asymmetry and bifurcation angle asymmetry. Which seems to indicate that area ratio is more important than bifurcation angle in carotid stenosis. Spelde et al. [13] in a post-mortem examination of 100 carotid bifurcations measured the total area ratios. The calculated mean value of the bifurcation area ratio for the 60 normal carotids was 1.47 , and the mean value for the 40 diseased bifurcation was 0.99 . They also found no difference in the mean values between right and left carotids. Perktold and Resch [14] studied the flow into a carotid bifurcation and showed that small variations in the curvature of the sinus wall can change their results. Perktold et al. [15] further investigated the influence of the bifurcation angle on the flow into a carotid bifurcation. They found that the flow separation occurred over the major part of the cycle at larger angles, while at smaller angles it only occurred during the systolic phase of the particular waveform they used.

Tadjfar et al. [16-18] using the direct simulation of the governing equations discuss the development of a finite volume flow solver for the study of flow in the human cardiovascular system. This code was validated and verified by comparing with results of flows into bends and other biological flows. Further, they studied the steady and pulsatile flow into a $45^{\circ}$ branched tube. Smith et al. [19] discuss the flow into various branching tubes by theoretical modeling and direct numerical simulation. Tadjfar and Smith [20] by comparing the results of the slender flow theory with direct numerical simulation investigate the flow inside of a circular cylinder that splits into two half cylinders at a bifurcation junction. 
Here, we want to concentrate on the influence of the geometrical parameters: area ratio and bifurcation angle on the flow into a model three-dimensional bifurcation. In order to minimize the number of possible variations, we maintain daughter tube symmetry in terms of diameter and bifurcation angle. We can also study the influence of the convective acceleration on the flow by changing the inlet flow Reynolds number. But, we avoid the influence of the unsteady acceleration and the waveform shape by considering steady flow. We assume the blood vessels walls are rigid. The mother vessel of the branch is a circular tube that bifurcates into two daughter tubes of equal size. The mother tube opens in the middle into two diverging daughter tubes of circular cross sections (see Figure 1). The flow in a large number of geometric variations of our branch model is numerically simulated. The models at several branch area ratios, defined as the ratio of total cross sectional area of the daughter tubes to the cross sectional area of the mother tube, in the range of $0.4 \leq A R \leq 2.0$ are considered. In the above range of area ratios, we also create various versions within a range of branch opening half-angle of $\pi / 25 \leq \theta \leq \pi / 4$, defined as the angle between the axis of the mother tube and that of any of the daughter tubes (they are the same for symmetric bifurcations studied here). The flow in all the above models is calculated for the cases with the inlet flow Reynolds number of 500 and 1000 . In some of the above models, the flow is also simulated at $\operatorname{Re}=250$ and $\mathrm{Re}=2000$. The influence of the area ratio, inlet flow Reynolds number, and the branch opening half-angle on the distribution of axial shear stress and pressure along the branch walls and the formation of flow separation in the vicinity of the branch bifurcation are investigated.

\section{Numerical Method}

It is assumed that the fluid is homogeneous, incompressible and Newtonian. The governing equations are the 3-D incompressible, unsteady Navier-Stokes equations in the strong conservative form. The equations are non-dimensionalized and written in a generalized curvilinear coordinate system, such that:

$$
\int_{V(t)} \frac{\partial \overline{\mathrm{q}}}{\partial \tau} d V+\frac{\partial}{\partial t} \int_{V(t)} \overline{\mathrm{Q}} d V+\oint_{S(t)}\left(\overline{\overline{\mathrm{f}}}-\overline{\mathrm{Q}} \overline{\mathrm{v}}_{\mathrm{g}}\right) \cdot \hat{\mathrm{n}} d S=0
$$

Where $\overline{\overline{\mathrm{f}}}=\left(\overline{\mathrm{F}}+\overline{\mathrm{F}}_{\mathbf{v}}, \overline{\mathrm{G}}+\overline{\mathrm{G}}_{\mathbf{v}}, \overline{\mathrm{H}}+\overline{\mathrm{H}}_{\mathbf{v}}\right)$ and,

$$
\begin{aligned}
& \overline{\mathrm{Q}}=\left[\begin{array}{c}
u \\
v \\
w \\
0
\end{array}\right], \quad \overline{\mathrm{q}}=\left[\begin{array}{c}
u \\
v \\
w \\
p
\end{array}\right], \quad \overline{\mathrm{F}}=\left[\begin{array}{c}
u^{2}+p \\
u v \\
u w \\
\beta u
\end{array}\right], \quad \overline{\mathrm{G}}=\left[\begin{array}{c}
v u \\
v^{2}+p \\
v w \\
\beta v
\end{array}\right], \quad \overline{\mathrm{H}}=\left[\begin{array}{c}
w u \\
w v \\
w^{2}+p \\
\beta w
\end{array}\right],
\end{aligned}
$$

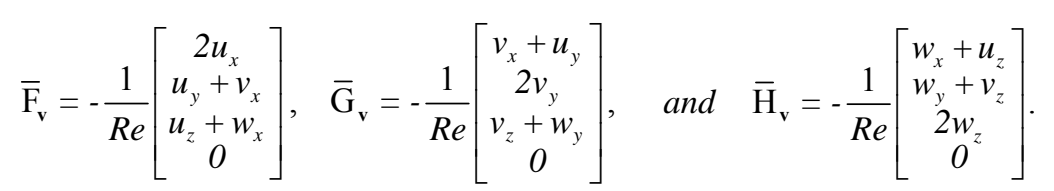


In the preceding equations $\mathrm{p}$ is pressure, and $(u, v, w)$ are respective velocity components in the physical Cartesian coordinate system: $(x, y, z)$. Reynolds number of the flow is defined based on the reference velocity and tube diameter as $\operatorname{Re}=\mathrm{U}_{\text {ref }} \mathrm{D} / \mathrm{v}$. Here, $\mathrm{t}$ denotes the real physical time, $\tau$ is the pseudo time, and $\beta$ is the pseudo-compressibility coefficient. $\quad \mathrm{V}(t)$ is the time varying volume of the cell. $S(\mathrm{t})$ denotes the surface of the control volume, and $\hat{n}$ is the outward unit normal vector at the surface of the control volume, where $\overline{\mathrm{v}}_{\mathrm{g}}$ is the local velocity of the moving control surface. Note that the term $\overline{\mathrm{q}}$ associated with the pseudo time is designed for an inner sub-iteration at each physical time step, and will vanish when the divergence of velocity is driven to zero so as to satisfy the equation of continuity.

For a structured boundary-fitted computational coordinate system: $(\xi, \eta, \zeta)$ and a cell-centered finite volume formulation, we can write equation (1) in a semi-discrete form for each cell centered at point (i,j, k):

$$
S t \frac{\partial}{\partial t}[V \overline{\mathrm{Q}}]_{\mathrm{ijk}}+\overline{\mathrm{R}}_{\mathrm{ijk}}+S t V_{\mathrm{ijk}}\left(\frac{\partial \overline{\mathrm{q}}}{\partial \tau}\right)_{\mathrm{ijk}}=0 .
$$

Where the steady state residual is given by:

$$
\begin{aligned}
& \overline{\mathrm{R}}_{i j k}=(\hat{\overline{\mathrm{F}}}+\hat{\overline{\mathrm{F}}})_{\mathrm{i}+\frac{1}{2}, \mathrm{j}, \mathrm{k}}-(\hat{\overline{\mathrm{F}}}+\hat{\overline{\mathrm{F}}})_{\mathrm{i}-\frac{1}{2}, \mathrm{j}, \mathrm{k}}+\left(\hat{\overline{\mathrm{G}}}+\hat{\overline{\mathrm{G}}}_{\mathrm{v}}\right)_{\mathrm{i}, \mathrm{j}+\frac{1}{2}, \mathrm{k}} \\
& -\left(\hat{\overline{\mathrm{G}}}+\hat{\overline{\mathrm{G}}}_{\mathrm{v}}\right)_{\mathrm{i}, \mathrm{j}-\frac{1}{2}, \mathrm{k}}+\left(\hat{\overline{\mathrm{H}}}+\hat{\overline{\mathrm{H}}}_{\mathrm{v}}\right)_{\mathrm{i}, \mathrm{j}, \mathrm{k}+\frac{1}{2}}-\left(\hat{\overline{\mathrm{H}}}+\hat{\overline{\mathrm{H}}}_{\mathrm{v}}\right)_{\mathrm{i}, \mathrm{j}, \mathrm{k}-\frac{1}{2}} .
\end{aligned}
$$

And the modified flux terms are defined as:

$$
\begin{aligned}
& \hat{\overline{\mathrm{F}}}+\hat{\overline{\mathrm{F}}}_{\mathrm{v}}=\left(\overline{\overline{\mathrm{f}}}-S t \overline{\mathrm{Q}} \overline{\mathrm{v}}_{\mathrm{g}}\right) \cdot \overline{\mathrm{S}}_{n}^{\xi} \\
& \hat{\overline{\mathrm{G}}}+\hat{\overline{\mathrm{G}}}_{\mathrm{v}}=\left(\overline{\overline{\mathrm{f}}}-S t \overline{\mathrm{Q}} \overline{\mathrm{v}}_{\mathrm{g}}\right) \cdot \overline{\mathrm{S}}_{n}^{\eta} \\
& \hat{\overline{\mathrm{H}}}+\hat{\overline{\mathrm{H}}}_{\mathrm{v}}=\left(\overline{\overline{\mathrm{f}}}-S t \overline{\mathrm{Q}} \overline{\mathrm{v}}_{\mathrm{g}}\right) \cdot \overline{\mathrm{S}}_{n}^{\zeta} .
\end{aligned}
$$

The normal-area vector in the $\xi$-direction is:

$$
\bar{S}_{n}^{\xi}=\left[S_{n x}^{\xi}, S_{n y}^{\xi}, S_{n z}^{\xi}\right]
$$

In the above formulation the flow Strouhal number is defined as $S t=D /\left(U_{r e f} t_{r e f}\right)$. The physical time derivatives are differenced using the second order trapezoidal implicit method, while the first order Euler implicit differencing is used on the pseudo-time derivatives. The inviscid fluxes are differenced using third-order upwind implementation of Roe's flux-difference split averaging technique. Second order central differencing is used on the viscous fluxes. To maintain second order spatial accuracy a special treatment at the boundaries is required.

Flow Solver. All the numerical simulations are achieved by using the fast, parallel, and time accurate solver of Tadjfar et al. [16-18]. The solver is capable of dealing with moving boundaries and moving grids. It is designed to 
handle complex, three-dimensional vascular systems. The computational domain is divided into multiple block subdomains. At each cross section the plane is divided into twelve sub-zones to allow flexibility for handling complex geometries and, if needed, appropriate parallel data partitioning. Modeling of the human vascular flow could require massive amounts of computation. Accurately simulating a large three-dimensional model of a patient's vascular system requires high-speed hardware with large memory space. It is now widely recognized that the parallel processing is the platform for processing such systems. Communication between the subdomains of the flow is implemented using MPI message-passing library. The code is capable of being implemented on both shared and/or distributed memory architectures.

Boundary Conditions. At the inlet of the mother tube a fully developed Poiseuille flow is assumed for the velocities, and the condition $\partial_{S S}(P)=0$ is imposed on the pressure, where $\mathrm{s}$ is the local streamwise direction along the centerline of each tube. We also define a local normal direction on the wall, $\mathrm{n}$, which is always normal to the local wall and defined inwardly positive towards the center of the tubes. Both $\mathrm{s}$ and $\mathrm{n}$ are nondimensionalized by the main tube diameter. At the outlet of each daughter tube the pressure is prescribed as $P=0$, and for the velocities the condition $\partial_{s s}(u, v, w)=0$ is imposed. Along the rigid walls the no slip condition is assumed $(u, v, w)=0$. All the dimensions are nondimensionalized by the mother tube diameter, MD, and all the velocities are nondimensionalized by the mean (bulk) velocity of the inlet profile.

Grid Independence. Computational grids for each of the various cases considered are generated based on the branch model chosen here. Three grids of different grid density and size were considered: 1) Grid1 extends 15 MD upstream and $10 \mathrm{MD}$ downstream of the bifurcation in each daughter tube. This grid comprises of 55,230 cells. In the mother tube, we use a $42 \times 51 \times 15$ grid, which implies using 42 cells in the tube axial direction by 51 cells in the azimuthal direction and 15 cells in the radial direction at each cross section of the tube. Similarly in each of the daughter tubes, we use a $33 \times 35 \times 10$ grid. Appropriate grid stretching near the walls in the radial direction and near the bifurcation region in the axial direction is implemented. 2) Grid2 is a lower density version of Grid 1 with 41,280 cells. We use a $32 \times 51 \times 15$ grid in the mother tube and a $24 \times 35 \times 10$ grid in each of the daughter tubes. 3) Grid3 is a much shorter grid that extends only 5 MD upstream and downstream from the bifurcation comprising of 57,375 cells. For this high-density grid we use a $25 \times 51 \times 15$ grid in the mother tube and in each of the daughter tubes. This grid for the case with $\theta=\pi / 8$ is shown in figure 1 .

All the simulation cases were repeated for both Grid1 and Grid3 and some cases were also tested with Grid2. The results were close in all cases for most of the flow, but for the peak values occurring in a very small section of the walls very close to the bifurcation region. The maximum difference in the peak values of the wall shear and wall pressure 
between all the grids considered here was less than $6 \%$. The centerline pressure along the mother tube (MT) and left daughter tube (LDT) for the case of $\mathrm{AR}=1.0$ and $\theta=\pi / 8$ at $\mathrm{Re}=500$ for all three grids are shown in figure $2 \mathrm{a}$ (The worst comparison location). The normal gradient of the axial velocity, a measure of wall shear, running along a line from the center of the right wall in the mother tube and along the center of the outer wall in the right daughter tube (RDT) is shown in figure $2 \mathrm{~b}$. Any of the three grids produces grid-independent numerical results. All the charts presented here are based on the Grid1 simulation results and all the visualization figures are presented on Grid3.

\section{Flow into An Arterial Branch Model}

A systematic study of the flow into a three dimensional model of an arterial branch is performed. The flow into an arterial branch with various values of the total area ratio and the opening angle of the bifurcation at several Reynolds numbers are considered. Due to the enormity of the task, we limit this study to the case of symmetric bifurcations. However, we solve over a full grid comprising of the entire branch. Hence, we are capable of solving for any asymmetry in shape, size, branch angle, and any tube differences in general in the future. We consider the role of the convective acceleration on the flow as shown in different Reynolds number simulations. But, the influence of the unsteady acceleration on this flow, as controlled by the shape and type of the flow waveform, is currently underway and will be reported in another paper. All the computational results presented here are achieved by allowing the residual on velocities to drop to $10^{-10}$ and the residual on mass conservation drop to $10^{-7}$.

Several lines along the axial direction in the tubes are chosen to present the distribution of flow variables. MT centerline is defined as the centerline of the tube along the axial direction in the mother tube. MT right line is defined as the line along the wall in the middle of the right wall (3 o'clock looking into the tube from downstream). MT top line is defined as the line along the wall in the middle of the top wall (12 o'clock looking into the tube from downstream). RDT centerline is defined as the centerline of the tube along the axial direction in the right daughter tube. RDT outer line is defined as the line along the wall in the middle of the outer wall ( 3 o'clock looking into the tube from downstream) which is the continuation on MT right line into the RDT. RDT inner line is defined as the line along the wall in the middle of the inner wall (9 o'clock looking into the tube from downstream) which is the line in the middle of the new dividing wall started after the bifurcation.

Flow Into A $45^{\circ}$ Branch. First, we consider the flow for a case in the middle of our parametric range of a $45^{\circ}$ bifurcation $(\theta=\pi / 8)$ with an area ratio, $\mathrm{AR}=1$, as shown in figure 1 . This implies that the whole flow in the mother tube will not see any flow expansion since the total cross sectional area remains constant through the bifurcation. In fact, there is a little flow acceleration due to the formation of new boundary layers in the newly formed inner walls of the 
bifurcation in the daughter tubes. The contour plots of streamwise velocity of the steady flow into this bifurcation at $\mathrm{Re}=$ 500 are presented in figure 3 (Grid3). The inlet velocity profile of a Poiseuille flow is presented in figure $3 a$. In the mother tube, near the bifurcation at $0.1 \mathrm{MD}$ to the junction, we can see the influence of the oncoming bifurcation walls. The high velocity core of the flow is showing a double peak as if it is getting ready to divide into the daughter tubes (figure 3b). In the daughter tubes, near the entrance at 0.3MD after the junction, the high momentum fluid core is pushed to the inner walls (towards the bifurcation center) of the tube (see figure $3 \mathrm{c}$ ). This is due to the position of the high velocity fluid core in the mother tube. This would result in a local region of high pressure and high wall shear stress at the inner walls of the daughter tubes. The local high-pressure region interacts with the surrounding boundary layers to set up the wellknown secondary flow to take the fluid back towards the outer side along the tube walls. Hence, the low momentum fluids near the outer walls are pushed into the core of the daughter tubes. This can clearly be seen in the figure $3 \mathrm{~d}$, where the streamwise velocity contours are presented at $1 \mathrm{MD}$ after the junction.

Such secondary flow patterns are typical of the flow at tube bends. However, in a branch the secondary flow pattern is enhanced. This is due to the position of the incoming high momentum core of the mother tube, which is already located near the inner walls of the daughter tubes prior to bifurcation. The influence of this secondary flow can be seen all the way to the outlet of the daughter tubes. Figure $3 \mathrm{e}$ shows the streamwise velocity contours at $2.5 \mathrm{MD}$ in the daughter tubes, where the formation of the typical crescent-shape contours is evident. There is a lower velocity core in the middle of the tubes with the higher velocity fluids near the walls of the tube creating higher shear stresses at the wall. However, by the action of viscous diffusion the velocity gradients will gradually dissipate. This is clearly evident in the velocity contours at $5 \mathrm{MD}$ (at the outlet of Grid3) in the daughter tubes presented in figure $3 \mathrm{f}$. The same thing can be seen in the results for the longer Grid1, where the viscous diffusion has further eroded the secondary flow by 10MD at its outlet.

The Influence of Bifurcation Angle. Here, we investigate the influence of the bifurcation angle on the flow. We consider a physiologically relevant range of branch opening half-angle of $\pi / 25 \leq \theta \leq \pi / 4$ which corresponds to the bifurcation angles of roughly $14^{\circ}$ to $90^{\circ}$. To fix other parameters and concentrate on the bifurcation angle only, we present data for the flow of the branches with $\mathrm{AR}=1$ at $\mathrm{Re}=500$. Figure 4 presents the pressure distributions along the centerlines of MT and RDT at various angles. The pressure drop is mostly linear except in the region from 1 MD upstream to $2 \mathrm{MD}$ downstream of the bifurcation. In the MT centerline, near the bifurcation, the pressure rises due to the approaching stagnation region of the dividing surface. In the beginning of the RDT centerline, the flow moves away from the vicinity of the stagnation region, in the flow divider surface in between the two daughter tubes, and continues with the pressure drop. As the branch half-angle is increased from $\pi / 25$ to $\pi / 4$, the non-dimensional pressure at $\mathrm{s}=1$ is increased 
from 2.45 to 2.8 , indicating a pressure increase of about $14 \%$ to adjust for the extra losses due to the enhanced secondary flow patterns.

Figure 5 presents the normal gradient of the axial velocity, a measure of wall shear, along the right lines of MT and their extension along the outer lines of RDT at various angles. The velocity gradients are normalized by their fully developed values in a Poiseuille flow. The entire domain is non-dimensionalized by the MT parameters, which results in the Poiseuille flow velocity gradient value of 1 in the mother tube and $\sqrt{2}$ in the daughter tubes for the case with $\mathrm{AR}=1$. We can observe two emerging trends in this figure. In the outer wall of RDT, we can see a tendency towards separation near the bifurcation region as $\theta$ is increased. In fact, at higher values of Re or AR there would be flow separation as will be shown later. Just before the bifurcation region along the right wall of MT, as $\theta$ is increased, we can see a gradual increase in the peak value of the wall gradient from 1.12 for the case with $\theta=\pi / 25$ to 1.58 for the case with $\theta=\pi / 4$ (41\% increase). There is a short upstream influence and the Poiseuille flow still exists past the location at $\mathrm{s}=12$. There is a narrowing of the upstream influence at higher bifurcation angles, which we will discuss more about it later.

Figure 6 gives the distribution of axial wall shear along the inner line of RDT. Immediately near the bifurcation region, there are high values of wall shear. For $\theta=\pi / 25$ branch the initial wall gradient is $8.4(600 \%$ of the Poiseuille value), as $\theta$ is increased this initial value drops to 5.4 for the case with $\theta=\pi / 4$. However, this initial peak drops faster for lower angles as we move away from the bifurcation region, resulting in higher values of shear at higher branch angle. The axial wall shear distributions along the top line of MT are presented in figure 7 . Very close to the bifurcation, the wall shear values drop as $\theta$ is increased. This leads to separation at the top wall of MT, just before the bifurcation, for the case with $\theta=\pi / 4$ even at this Reynolds number $(\operatorname{Re}=500)$. There is also a second trough in the shear value further upstream and a tendency for the spreading of the upstream influence as branch angle is reduced. This counter intuitive behavior is probably due to the particular way of smoothing (geometric transition) from MT to the daughter tubes, that is chosen here and is case specific. We will talk about this more in the discussion section.

The Influence of Reynolds Number. We can study the role of the convective acceleration on the flow by varying the flow Reynolds number. This is another important factor that influences the formation of separation near the branching region. By keeping the geometry and the inlet profile the same, increasing the flow Reynolds number is equivalent to lowering viscosity of the fluid. Pressure distributions along the centerlines of MT and RDT at different Reynolds numbers for the cases with $\theta=\pi / 8$ and $\mathrm{AR}=1.0$ are given in figure 8 . The pressure gradient is mostly linear except near the bifurcation. As Re is decreased from 2000 to 250 , the non-dimensional pressure at $\mathrm{s}=1$ goes from 0.86 to 4.78 , indicating a pressure increase of about $560 \%$. The value of the peak in the pressure distribution in the mother tube, near the stagnation region of the dividing surface, goes from 1.41 to 3.96 (280\% increase). 
Figure 9 presents the axial wall shear along the right lines of MT and their extension along the outer lines of RDT at several Reynolds numbers for the cases with $\theta=\pi / 8$ and $\mathrm{AR}=1.3$. As mentioned previously, at higher values of Re there is a region of back flow (separation) at the bifurcation area. The upstream influence of Reynolds number in the peak values of wall shear prior to the bifurcation is minimal. The size of the separation region and the strength of the back flow increase with the Reynolds number. Hence, a much stronger secondary flow is created as Re is increased. The viscous damping of the induced secondary flow and its effect on the main flow require a much longer distance at higher Re values. The distributions of axial wall-shear along the inner line of RDT are given in Figure 10. Immediately after the bifurcation there are high values of wall shear, which drops rapidly as the flow moves downstream. For the flow at $\mathrm{Re}=250$, we can see a return to the fully developed profile and the non-dimensional shear value of $\sqrt{2} /(1.3)^{3 / 2}$ in the daughter tubes for the case with $\mathrm{AR}=1.3$.

The Influence of Area Ratio AR. Another important geometric factor that influences the flow in a branch is the area ratio. Pressure distributions along the centerlines of MT and RDT at several area ratio values are presented in figure 11 for the cases with $\theta=\pi / 8$ and $\mathrm{Re}=500$. The pressure gradient is mostly linear except near the bifurcation. As AR is increased from 0.4 to 2.0 , the non-dimensional pressure at $\mathrm{s}=1$ drops nonlinearly from 15.2 to 0.98 . Figure 12 presents the axial wall shear along the right lines of MT and their extensions along the outer lines of RDT at several values of the area ratio for the cases with $\theta=\pi / 8$ and $\operatorname{Re}=1000$. The upstream influence of the bifurcation is limited to less than two diameters in the MT. At higher values of AR, prior to the bifurcation in the mother vessel, the wall-shear values drop to an eventual back flow region. For the case with $\mathrm{AR}=2.0$, this separation region is fairly large (almost $3 \mathrm{MD}$ in length). For the cases with area ratios that are low enough not to cause a flow separation, the wall shear initially increases in the MT to a peak value prior to the entrance of the daughter vessel. The peak shear values are $1.5,3$, and 7 times the fully developed values for the cases with $\mathrm{AR}=1.0,0.7$, and 0.4 respectively. For the case with $\mathrm{AR}=0.4$, we can see a second wall-shear peak value in the outer walls of the daughter vessel. All the wall shear values eventually reach their fully developed values further downstream in the daughter vessels as the viscous diffusion damps out the effect of the secondary flow on the velocity profiles.

The axial wall shear distributions along the top line of MT at different AR values for the cases with $\theta=\pi / 8$ and $\operatorname{Re}=$ 1000 are presented in figure 13. There seems to be an initial drop, followed by a peak and a final drop in the shear values just prior to the bifurcation. The wall shear values in the initial drop get lower, and the trough location moves downstream as $\mathrm{AR}$ is reduced. There exists a back flow region, $1 \mathrm{MD}$ in length and located at $\mathrm{s}=14.4$, for the case with $\mathrm{AR}=0.7$. The peak values in the wall shear also get higher, and the peak value location moves downstream as AR is reduced. The 
peak shear values are 1.6 and 2.9 times the fully developed values for the cases with $\mathrm{AR}=1.0,0.7$ respectively. The wall shear values at the final drop location, $\mathrm{s}=15$, go from almost zero to 1.3 as AR is reduced from 2.0 to 0.7.

The Back Flow Region Shape. Separation region is of particular importance in the study of arterial hemodynamics. These areas are generally associated with low momentum fluid particles. Hence, resulting in low wall shear stress regions and large particle residence times. The definition of separation regions in three-dimensional flows is complex. It involves defining singular points and the limiting streamlines in the flow field (see Tobak and Peake [21]). Here, we look at the back flow regions as a subset and indication of the complexity of the three-dimensional separation regions involved. In figure 14, we can see the influence of the area ratio on the shape of the back flow region. In the case, with $\mathrm{Re}=1000$ at the area ratio of $\mathrm{AR}=1.3$ (Fig. 14a) we can see a small region of back flow is present on the outer wall of the daughter branches. Increasing the area ratio to $\mathrm{AR}=1.6$ and $\mathrm{AR}=2.0$, enlarges the back flow region significantly, as can be seen in figures $14 \mathrm{~b}$ and $14 \mathrm{c}$ respectively. The shape of the back flow region is influenced by the magnitude of the Reynolds number of the flow. However, the influence of the Reynolds number is not as significant as the area ratio on the size of the separation region.

\section{Discussion}

We have investigated the steady flow into an arterial branch model in detail. In particular, we were interested in the role of the local geometric parameters of the bifurcation on the overall flow. Specially, how the changes in the bifurcation geometry influence the distribution of axial wall shear and pressure in the model. The patterns that developed were discussed in length in the sections presented previously. However, we shall summarize and make further comments below followed by some discussion of our results in relation to the clinical measurements and other works that were referred to in the introduction.

The high velocity core of the incoming profile has to turn into the daughter tubes in advance of the approaching stagnation region of the new dividing surface. In general, such upstream influences are short and abrupt, with a lot of the local details to be governed by the local wall curvatures and geometry. The pressure distribution is mostly dictated by the local geometry of the mother and daughter vessels (linear in our straight tube segments). The influence of the bifurcation on pressure is limited to mostly within 2 diameters upstream and downstream of the bifurcation dividing surfaces. The upstream influence of the bifurcation geometry on the velocity profiles and wall shear values is also limited to within 2 diameters. However, the downstream dissipation of the effects of the induced secondary flow, created at the bifurcation, on the velocity profiles and the wall shear distributions may last much longer and is strongly influenced by the Reynolds 
number of the incoming flow. Regions of flow separation, associated with slow flow, are of high interest in the vascular hemodynamics research. Two locations in the mother tube, middle of the top and bottom walls prior to the bifurcation, are prone to separation. This is due to existence of the stagnation regions of the dividing surface that initiates the newly formed inner walls of the daughter tubes. The outer walls of the daughter vessels at their entrance and their continuations into the mother vessel are also separation prone.

As the opening angle of the bifurcation is increased the possibility and severity of flow separation at the appropriate wall location increases. Increasing the bifurcation angle from $14^{\circ}$ to $90^{\circ}$, for the cases with $\mathrm{AR}=1$ and $\mathrm{Re}=500$, resulted in a $14 \%$ increase in the peak pressure value near the entrance of the MT (figure 4). Similarly, the peak value of the wall shear on the right wall of MT increased by $41 \%$ (figure 5). At this Reynolds number there was no flow separation in the outer walls of the daughter tubes and a small separation region was observed in the top and bottom walls of MT only for the highest bifurcation angle considered. The local details of the wall shear distributions, two troughs and one peak, on the top wall of MT presented in figure 7 are probably due to the local smoothing of the wall curvatures as the mother and daughter vessels join. Tadjfar and Smith [20] reported only one trough just prior to the bifurcation in the wall shear distribution. In their model, a straight cylinder that opens into two half cylinders with sharp corners, they have avoided wall curvature smoothing altogether. In figure 6 , the values of the axial wall shear along the inner wall of RDT approach the fully developed flow value asymptotically. At the end of our computational domain the influence of the secondary flow has not been damped out yet for all the branch angles considered at that Reynolds number. Interestingly, the initial peak at the entrance to RDT drops as $\theta$ is increased. This is due to the fact, that the flow has not yet turned far from the wall at the entrance and there is a large component of the normal velocity gradient as well as the axial wall gradient in the normal direction. Within a short distance, less than half diameter, the lines cross and we have higher axial shear values for higher bifurcation angle cases.

At higher values of the incoming flow Reynolds number, there is a higher possibility of formation of a separation region near the bifurcation and that the size of the separation region to be larger. Increasing the Reynolds number from 250 to 2000 , for the case with $\theta=\pi / 8$ and $A R=1.0$, resulted in a $560 \%$ drop in the pressure value at the entrance of $\mathrm{MT}$ (figure 8). This is less than a linear decrease of $800 \%$ as one would get for flow in a straight tube $\left(\partial_{s}(P)=-32 / \operatorname{Re}\right)$. This difference is probably caused by the extra losses due to the increase in the strength of the induced secondary flow. Similarly, for the case with $\theta=\pi / 8$ and $A R=1.3$, the non-dimensional pressure at $\mathrm{s}=1$ goes from 3.28 to 0.4 (820\% drop). However, here the area ratio is not one anymore and there is an over all increase in the cross sectional area in the daughter tubes. In figure 10, as we move away from the bifurcation the wall shear values drop rapidly in the inner walls of RDT. 
However, this drop is delayed at higher values of Re due to the narrowing of the daughter tubes at their inlet because of the local separation there. At $\operatorname{Re}=250$, the peak shear value is 6 and it's location is at the entrance of the RDT, $s=15$, but at $\operatorname{Re}=2000$ the peak value is $7.2(20 \%$ increase $)$ and it is located further downstream at $\mathrm{s}=15.3$.

Area ratio seems to have the largest influence on the flow within the physiologically relevant range of the parameters considered. Increasing the area ratio can lead to relatively large flow separation on the outer walls of RDT in the vicinity of the bifurcation region. As the area ratio is increased from 0.4 to 2.0 , the inlet pressure drops by $1,550 \%$ for the case with $\theta=\pi / 8$ and $\operatorname{Re}=500$ (figure 11 ). In fact, the relative pressure drop is almost inversely proportional to the square of the respective AR ratios. At higher values of the area ratio, there is none or a very small rise from the Poiseuille values in the axial wall shear before dropping to negative values (back flow) prior to the bifurcation junction on the right walls of MT (figure 12). For the case with $\mathrm{AR}=0.4$, there is a small initial drop in the value of wall shear that has moved further upstream and no upstream separation region exists. The peak value in the wall shear reaches 7.5 before it sharply drops to -1.1 at $\mathrm{s}=15$. This trend change in the wall shear behavior on the top wall of MT prior to the bifurcation requires further investigation. In figure 14, the projected cross sectional area of the back flow regions in the LDT and the projected span of the back flow regions in RDT are presented at three different area ratios. The resemblance of these shapes to the actual plaque shapes in arteries is amazing.

Our observations are in full agreement with the conclusions of Karino and Goldsmith [8] that the area ratio has a stronger influence on the flow than the bifurcation angle. However, theirs was based on observation of flow visualization patterns of side tubes branching from a main tube, here, we have quantitative values to compare the relative importance of each effect on a Y-shaped branch. The data of Sharp et al. [7] on occlusive disease seems to indicate a correlation between higher position and acute angles in aortic bifurcation and the disease. One has to be very careful to compare CFD results with arterial disease data. There are many factors we have not included in our analysis, the inlet wave form, wavereflection corrected unsteady outlet boundary conditions, wall dynamics, wall elasticity, wall material properties, etc. Any of these factors alone can change the flow. However, one can make some generalized observations with caution. We can not find any explanation for their observation. May be we need to include other factors in our analysis or we need to have more data on the area ratio of their cases as well. We seem to be in agreement with the observations of Friedman et al. $[10,11]$ on the positive correlation between large bifurcation angles and the possibility of plaque growth near the bifurcation and its location. Our results support the findings of Fisher and Fieman [12] that area ratio is a more influential parameter than the bifurcation angle on the formation of carotid stenosis. 


\section{REFERENCES}

[1] Caro, C.G., 1994, “Alterations of arterial hemodynamics Associated with risk factors for atherosclerosis induced by pharmacological means: implications for the development/management of atherosclerosis", In: Hosoda, S., Yaginuma, T., Sugawara, M., Taylor, M.G., Caro,C.G. (Eds.), Recent Progress in Cardiovascular Mechanics. Harwood Academi Publishers, Chur, Switzerland, pp.197 -213.

[2] Ku, D. N., 1997, "Blood flow in arteries”, Ann. Rev. of Fluid Mech. 29, pp. 399-434.

[3] Giddens, D. P., Zarins, C. K., Glagov, S., 1993, "The role of fluid mechanics in the localization and detection of atherosclerosis", ASME Journal of Biomechanical Engineering 115, pp. 588 -594.

[4] Pedley, T. J., 1995, "High Reynolds number flow in tubes of complex geometry with application to wall shear stress in arteries", In Biological Fluid Dynamics, 49th SEB symposium. The Society for Experimental Biology, pp. $219-241$.

[5] Ku, D. N., Giddens, D. P., Zarins, C. K., Glagov, S., 1985, "Pulsatile flow and atherosclerosis in the human carotid bifurcation", Arteriosclerosis, 5, pp. 293-302.

[6] Lou, Z., Yang, W., 1992, "Biofluid dynamics at arterial bifurcation”, Critical Reviews in Biomedical Engineering 19:455-493.

[7] Sharp, W. V., Donovan, D. L., Teague, P. C., and Mosteller, R. D., 1982, “Arterial occlusive disease: A function of vessel bifurcation angle”, Surgery, 91, pp. 680-685.

[8] Karino, T., and Goldsmith, H. L., 1985, "Particle flow behavior in models of branching vessels, II: effects of branching angle and diameter ratio on flow patterns”, Biorheology, 22, pp. 87-104

[9] Schulz, U. G. R., and Rothwell, P. M., 2001, "Major variation in carotid bifurcation anatomy: a possible risk factor for plaque development”, Stroke, 32, pp. 2522-2529.

[10] Friedman, M.H., and Zhaohua, D., 1998, "Relation between the structural asymmetry of coronary branch vessels and the angle at their origin", J. of Biomechanics, 31, pp. 273-278.

[11] Friedman, M.H., Baker, P.B., Zhaohua, D., and Kuban, B.D., 1996, "Relationship between the geometry and quantitative morphology of the left anterior descending coronary artery”, Atherosclerosis, 125, pp. 183-192

[12] Fisher, M., and Fieman, S., 1990, “Geometric factors of the bifurcation in carotid atherogenesis”, Stroke, 21, pp. 267271.

[13] Spelde, A. G. A., de Vosi, R. A. I., Hoogendam, I. J., and Heethaar, R. M., 1990, "Pathological-anatomical study concerning the geometry and atherosclerosis of the carotid bifurcation”, Eur. J. Vasc. Surg., 4, pp. 345-348. 
[14] Perktold K., and Resch M., 1990, "Numerical flow studies in human carotid artery bifurcations: basic discussion of the geometric factor in atherogenesis”, J. of Biomed. Eng., 12, pp. 111-123.

[15] Perktold K., Peter R.O., Resch M., Langs G., 1991, "Pulsatile non-Newtonian blood flow in three-dimensional carotid bifurcation models: a numerical study of flow phenomena under different bifurcation angles”, J. of Biomed. Eng., 13, pp. 507-515.

[16] Tadjfar, M., Yamaguchi, T., and Himeno, R., 2000, "Parallel Solver for Blood Flow in Human Vascular System”, Proceedings of IMECE-2000.

[17] Tadjfar, M. and Himeno, R., 2001, "Parallel, Multi-zone, Multi-block Solver To Study Arterial Branches in the Human Vascular System”, Proceedings of IMECE-2001, FED-24967.

[18] Tadjfar, M., and Himeno, R., 2002, “Time-Accurate, Parallel, Multi-Zone, Multi-Block Solver to Study the Human Cardio-Vascular System”, Biorheology, 39, pp. 379-384.

[19] Smith, F.T., Purvis, R., Dennis, S.C.R., Jones, M.A., Ovendon, N.C., and Tadjfar, M., 2003, "Fluid Flows Through Various Branching Tubes", Journal of Engineering Mathematics, Vol. 47, pp. 277-298.

[20] Tadjfar, M. and Smith, F.T., 2003, "Direct Simulation and Modeling of Basic Three-dimensional Bifurcation Tube Flows" Submitted to the Journal of Fluid Mech.

[21] Tobak, M., and Peake, D. J., 1982, “Topology of three-dimensional separated flows', Ann. Rev. of Fluid. Mech. 14, pp. 61-85. 


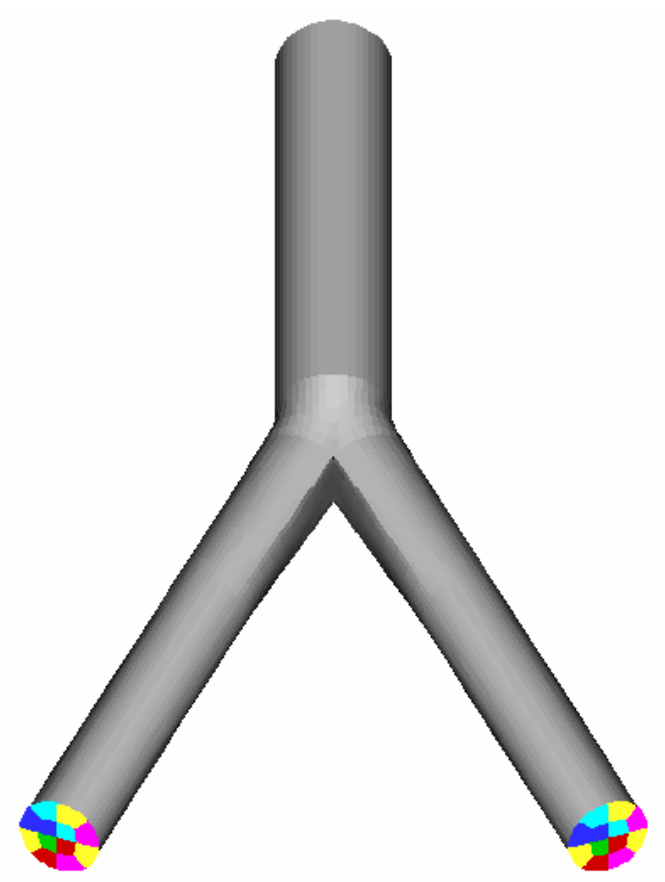

Fig. 1 Model of a $45^{\circ}$-Branched tube $(\theta=\pi / 8)$ with $A R=1$ on Grid3.

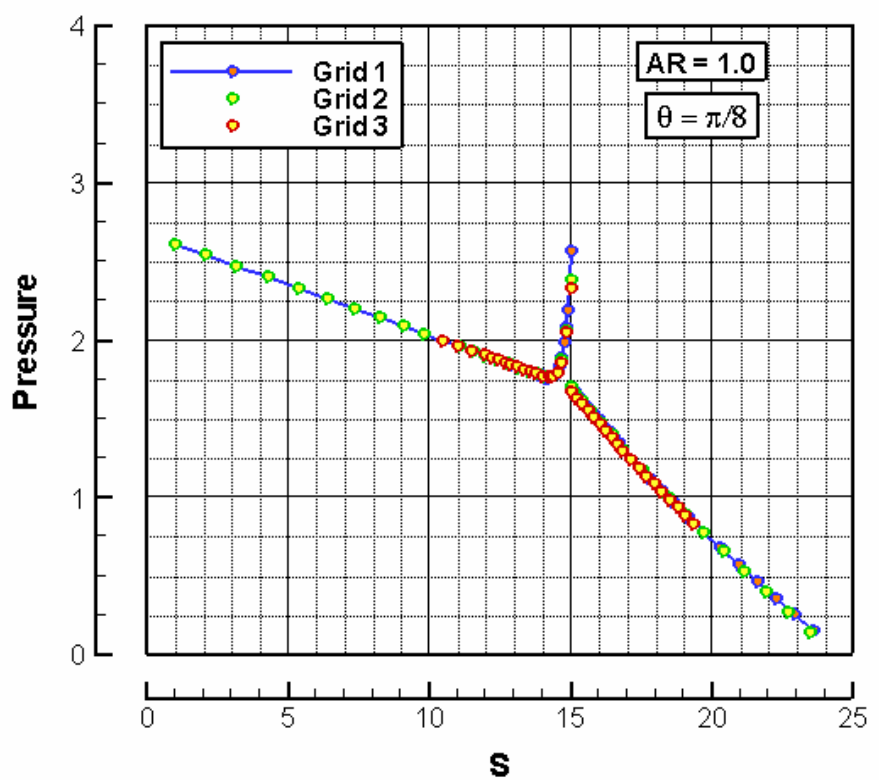

Fig. 2a Grid influence: pressure distribution along the centerline of the mother and the left daughter tube for the case $A R=1.0$ and $\theta=\pi / 8$ at $R e=500$. 


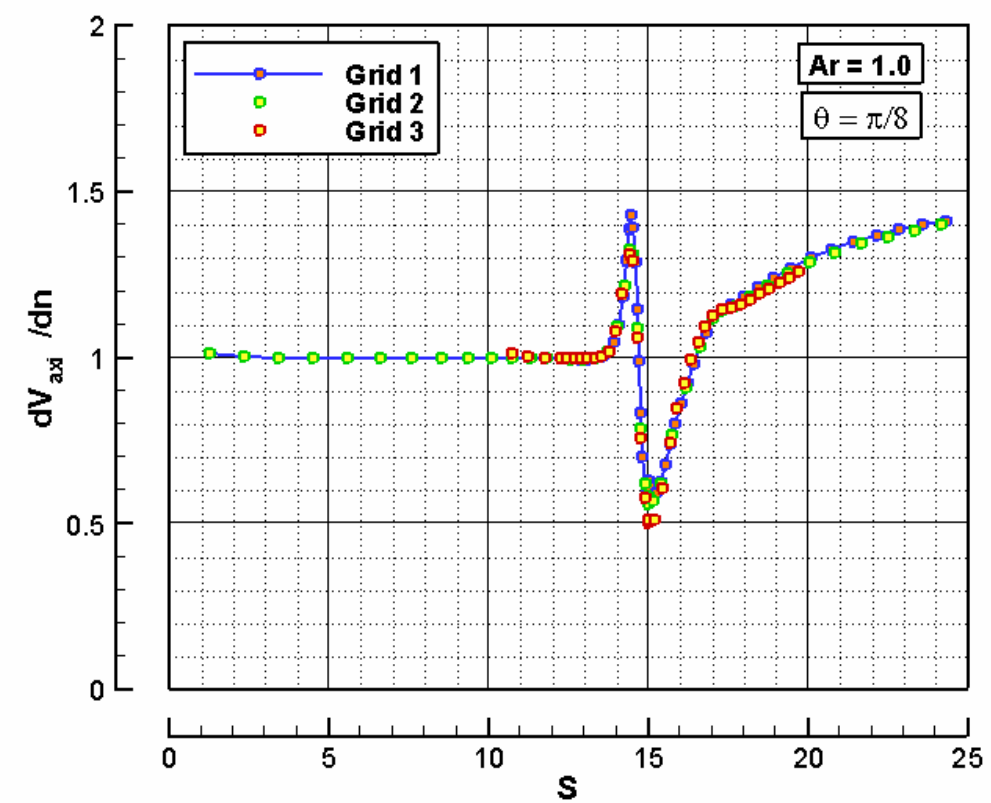

Fig. 2b Grid influence: Wall shear distribution along the right wall of the mother tube and

Outer wall of the right daughter tube for the case $A R=1.0$ and $\theta=\pi / 8$ at $R e=500$.

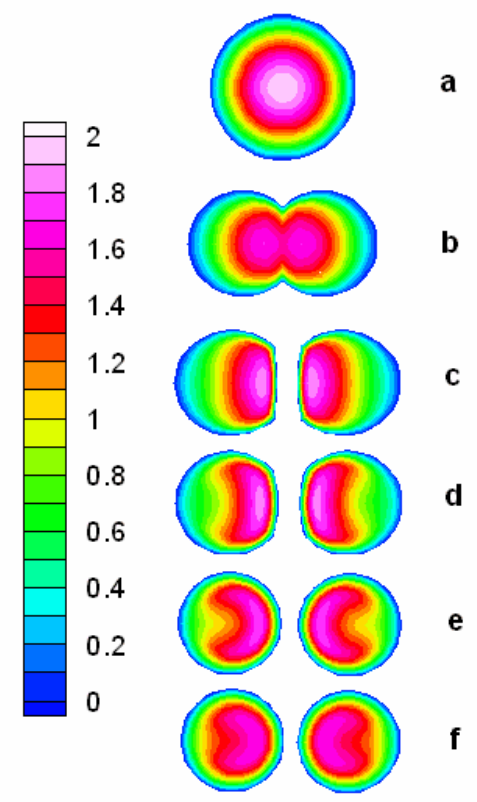

Fig. 3 Streamwise velocity contours $(A R=1.0$ and $\theta=\pi / 8$ at $R e=500)$ given in the mother tube at a) inlet and $b)$ $0.1 \mathrm{MD}$ to bifurcation, and in the daughter tubes at c) $0.3 \mathrm{MD}, \mathrm{d}$ ) $1 \mathrm{MD}, \mathrm{e}) 2.5 \mathrm{MD}$, and f) $5 \mathrm{MD}$ (outlet of Grid3) respectively. 


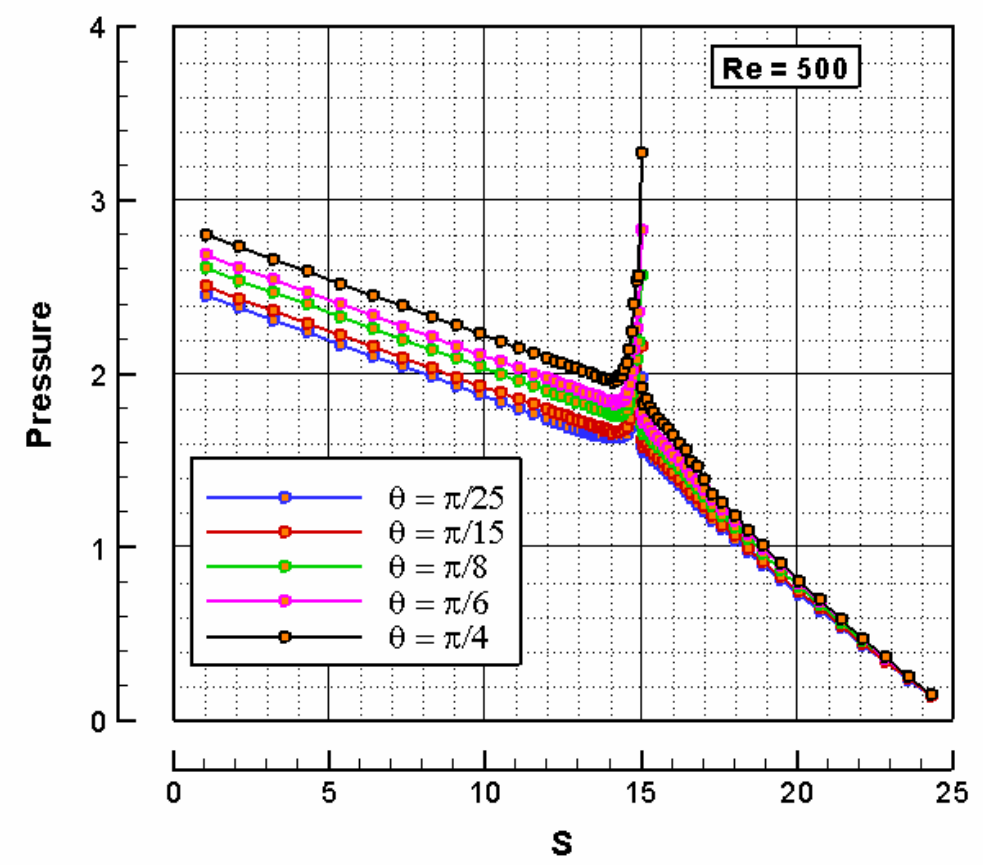

Fig. 4 Pressure distributions along the centerlines of $\mathrm{MT}$ and $\mathrm{RDT}$ for $\mathrm{AR}=\mathbf{1 . 0}$ at $\mathrm{Re}=\mathbf{5 0 0}$.

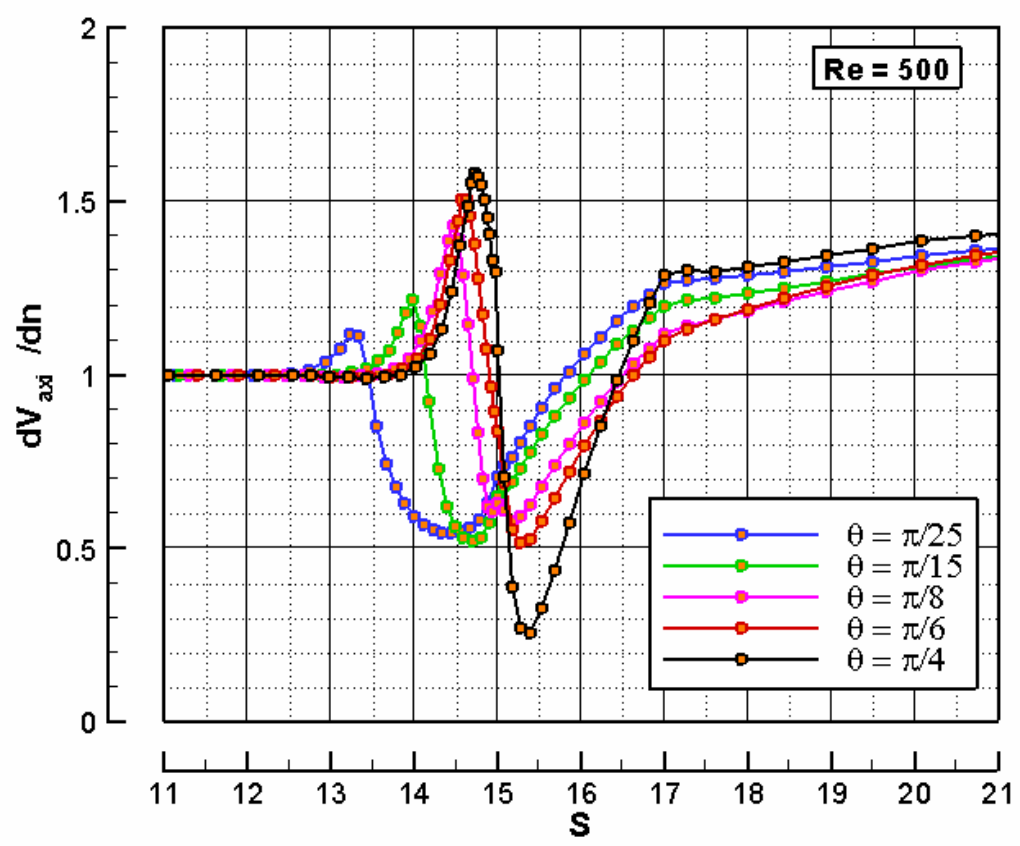

Fig. 5 Axial wall shear distributions along the right wall of $M T$ and the outer wall of $R D T$ for $A R=1.0$ at $R e=500$. 


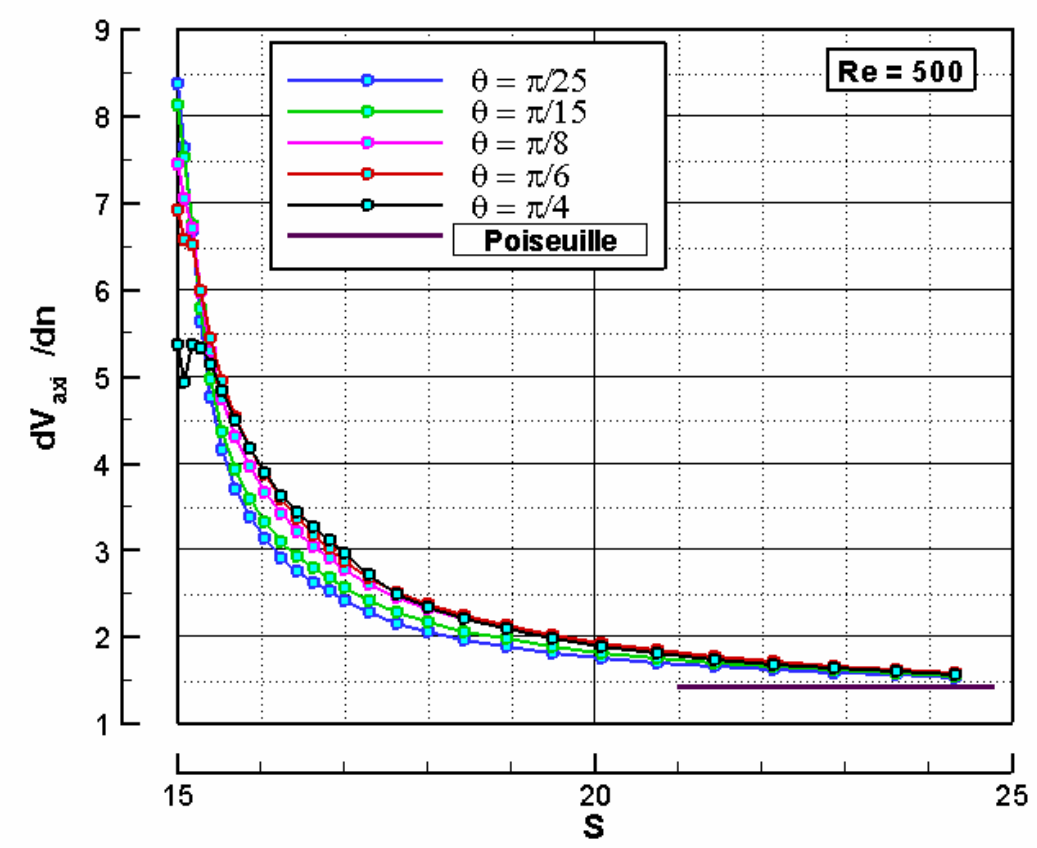

Fig. 6 Axial wall shear distributions along the inner wall of $R D T$ for $A R=1.0$ at $R e=500$.

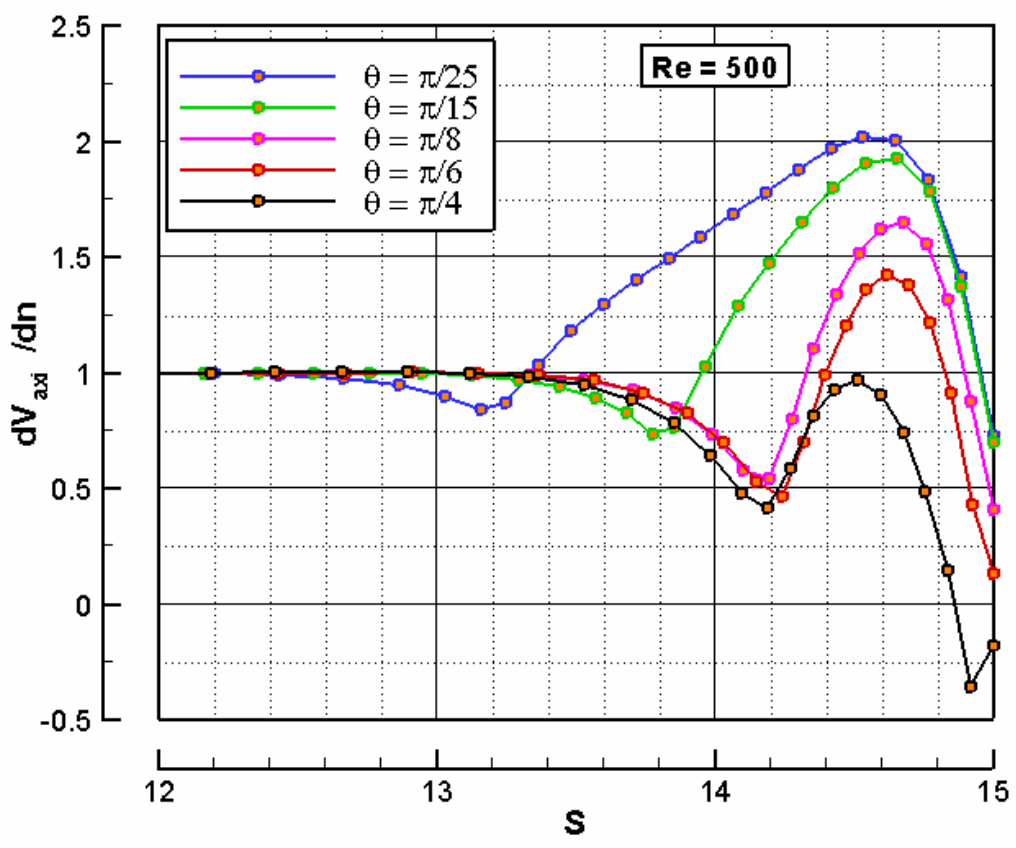

Fig. 7 Axial wall shear distributions along the top wall of $M T$ for $A R=1.0$ at $R e=500$. 


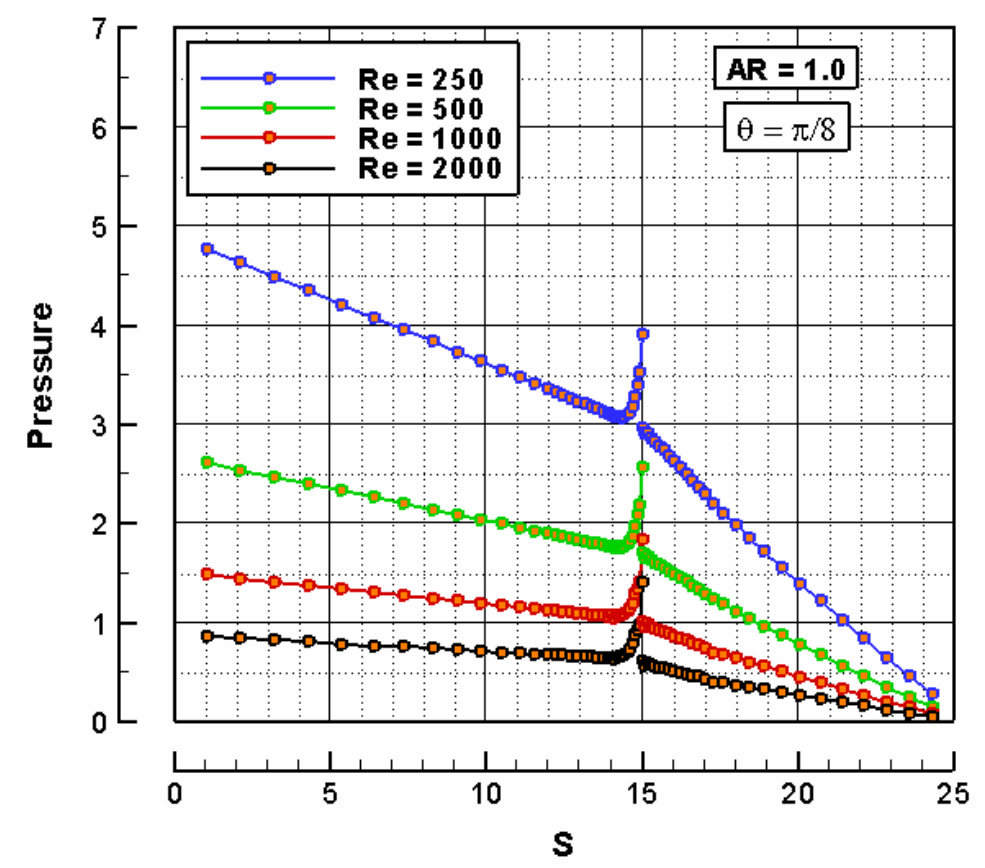

Fig. 8 Pressure distributions along the centerlines of MT and RDT for $\theta=\pi / 8$ and AR=1.0.

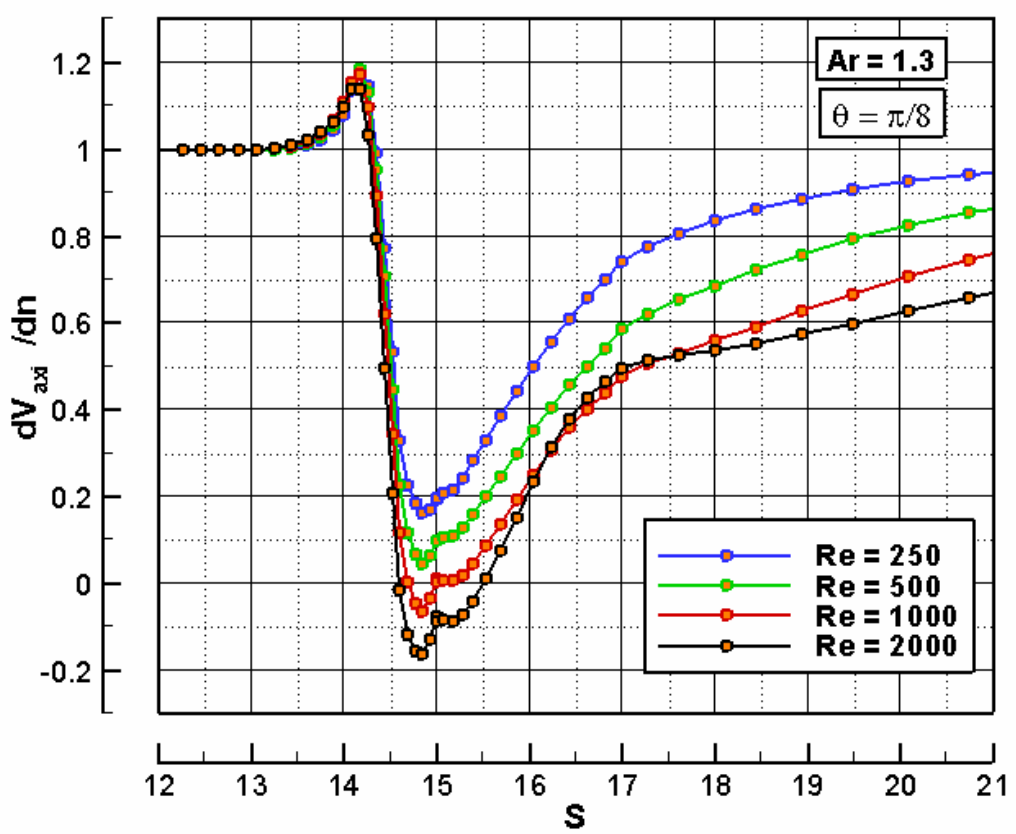

Fig. 9 Axial wall shear distributions along the right wall of MT and the outer wall of RDT for $\theta=\pi / 8$ and $A R=1.3$. 


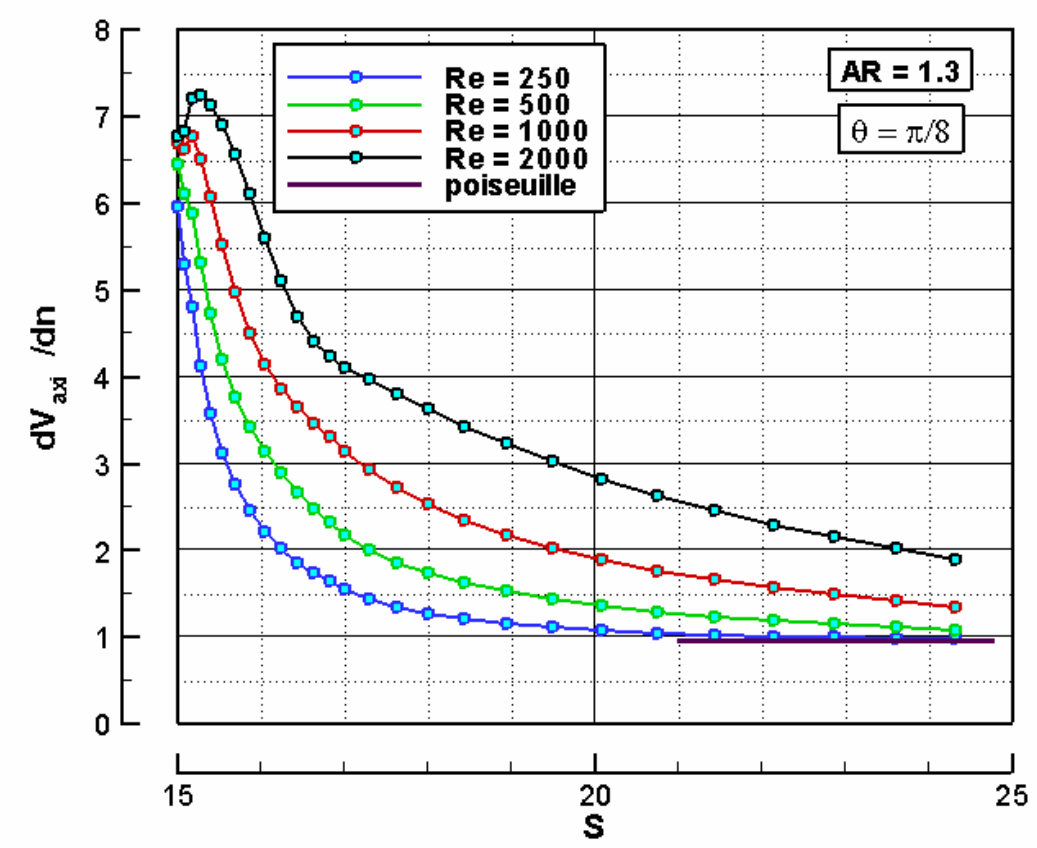

Fig. 10 Axial wall shear distributions along the inner wall of $R D T$ for $\theta=\pi / 8$ and $A R=1.3$.

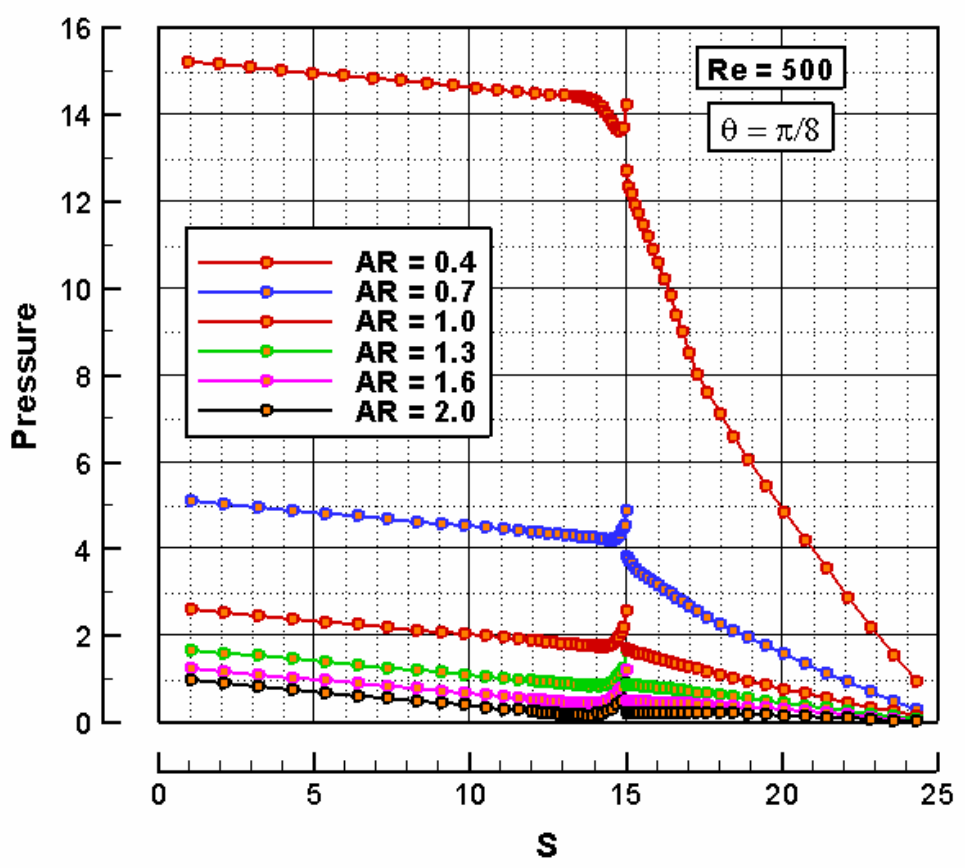

Fig. 11 Pressure distributions along the centerlines of $M T$ and $R D T$ for $\theta=\pi / 8$ and $\operatorname{Re}=500$. 


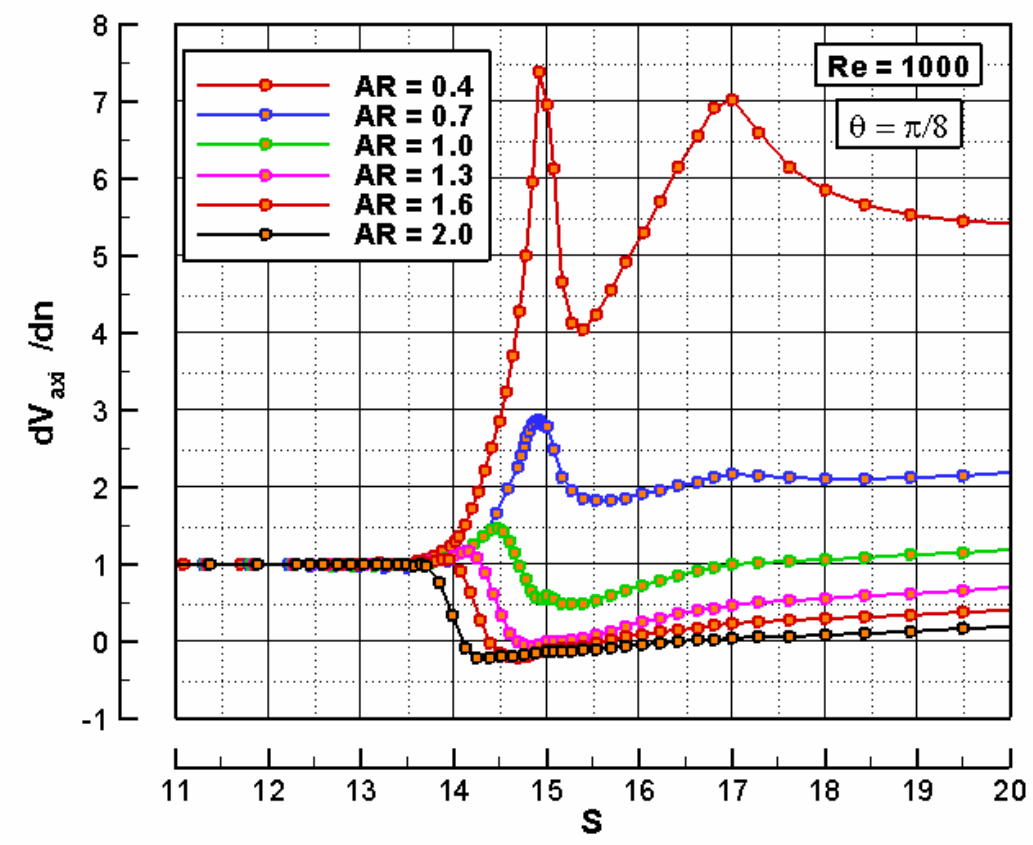

Fig. 12 Axial wall shear distributions along the right wall of MT and the outer wall of $\operatorname{RDT}$ for $\theta=\pi / 8$ and $\operatorname{Re}=$ 1000.

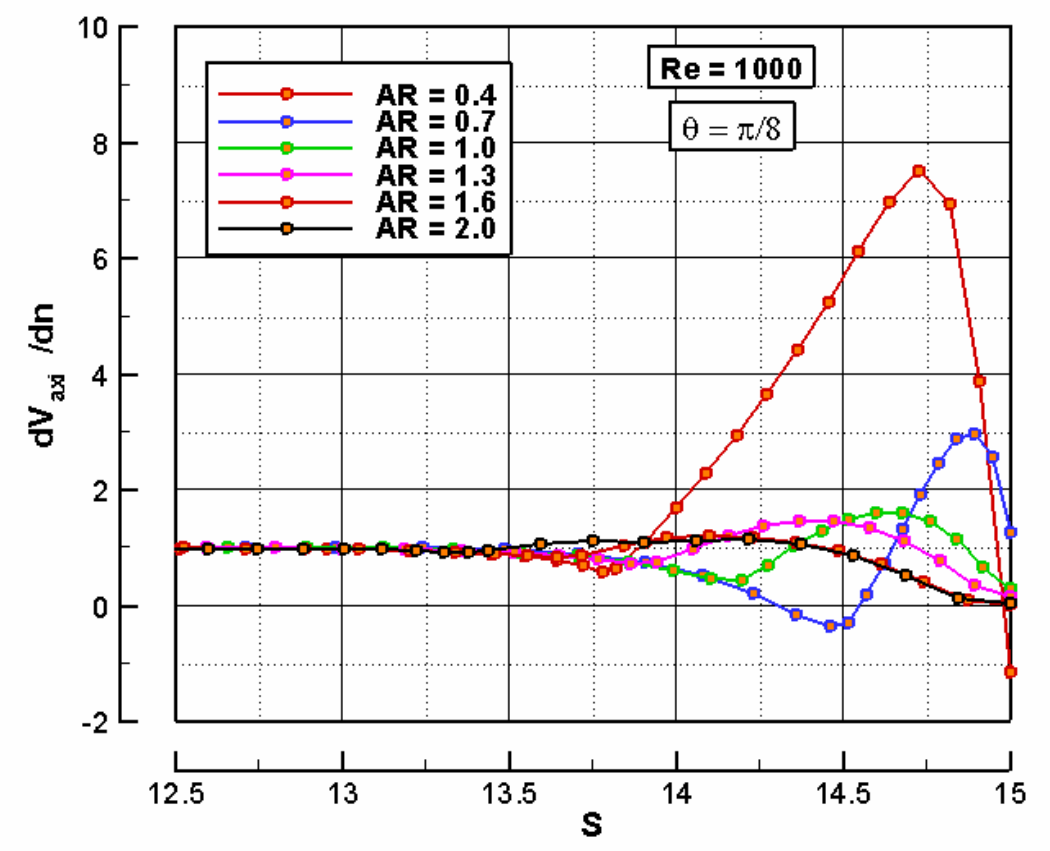

Fig. 13 Axial wall shear distributions along the top wall of $M T$ for $\theta=\pi / 8$ at $\operatorname{Re}=1000$. 

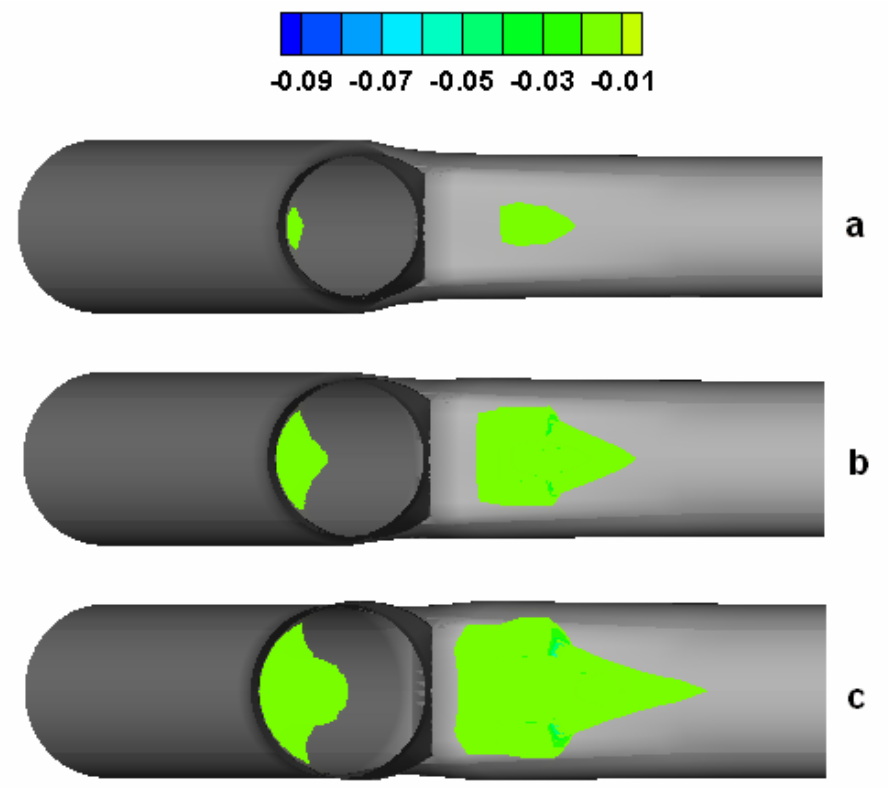

Fig. 14 Streamwise velocity surfaces (backflow part), looking down into the left daughter tube at $\operatorname{Re}=1000$ a) $\mathrm{AR}=1.3$, b) $\mathrm{AR}=1.6$, and c) $\mathrm{AR}=2.0$ (Grid3). 